\title{
Valvular Mapping of Rectus Abdominis and Latissimus Dorsi Muscles
}

\author{
Seyedhossein Aharinejad, Wolfgang Michlits, ${ }^{*}$ and Raymond M. Dunn ${ }^{* *}$ \\ * Center for Anatomy and Cell Biology, Vienna Medical University, A-1090 Vienna, Austria \\ ** Division of Plastic Surgery, University of Massachusettes, Worcester, MA 01655, USA
}

Free flaps obtained from the rectus abdominis and latissimus dorsi muscle are commonly used for reconstruction of musculo-cutaneous and cutaneous defects; e.g. venous insufficiencymediated ulcerations in the lower limb [1]. The ulcerations caused by venous insufficiency are often accompanied by pathologic hemodynamics and abnormal venous valves [2-3]. The aim of this study was to elucidate the venous valvular density in rectus abdominis and latissimus dorsi muscles, and thus to examine their benefit in reconstructive surgery [4].

The vasculature of 8 normal rectus abdominis and latissimus dorsi muscles was cast with Mercox ${ }^{\circledR}$ and biopsies were obtained from the upper, mid and lower segments of rectus abdominis (regions 1-3) as well as from the latissimus dorsi (regions 4-6) muscles [5]. Next, the microvalvular architecture of the samples was examined using scanning electron microscopy. The valvular and venous density was determined per $\mathrm{cm}^{2}$. The valvular index was calculated per $10 \mu \mathrm{m}$ venous length. Analysis of variance with the Bonferroni-t-test was used for statistical analysis.

Venous valves were observed in both muscles (Table 1). All valves observed were bicuspid (Fig. 1A) and were located exclusively just beneath the muscular fascia (Fig. 1B). In areas far from the fascia, no valves were found. The diameter of veins with valves ranged from $18 \mu \mathrm{m}$ to $243 \mu \mathrm{m}$. We compared the regions 1-3 and 4-6 and did not observe any significant difference in valvular index $(\mathrm{p}=0.750)$. However, we noticed a significantly higher valvular index in region 1 as compared to region $2(p<0.003)$ but not region $3(p=0.212)$. The valvular index was not different between regions 2 and $3(p=1.000)$. Regions 4 and 5 had a significantly higher valvular index as compared to region $6(\mathrm{p}<0.001, \mathrm{p}<0.003)$. The valvular index was not significantly different between regions 4 and 5 ( $p=1.000$; Fig. 2$)$.

This study indicates significantly different valvular densities within the different segments of both rectus abdominis and latissimus dorsi. The upper segment of the rectus abdominis might be preferred for reconstruction of cutaneous and musculo-cutaneous defects. Our data further suggest that the upper and the mid segments of the latissimus dorsi have a significantly higher valvular density than the lower segments of the muscle. Therefore, these segments might be preferred for reconstructive surgery. Furthermore, we demonstrate that all valves were located exclusively just beneath the muscular fascia, whereas no valves were found in deeper areas of the muscle, where only draining veins exist. Thus, musculo-cutaneous flaps might not be of a further benefit when compared to cutaneous flaps including the fascia with regard to their use for venous valve transfer in reconstructive surgery.

\section{References}

[1] D. Serafin et al., Plast. Reconstr. Surg. 66 (1980) 230.

[2] S. Aharinejad et al., Microcirculation. 8 (2001) 347.

[3] W. Mayer and H. Partsch, Curr. Probl. Dermatol. 27 (1999) 81.

[4] S.A. Taheri et al., J. Cardiovasc. Surg. 27 (1986) 163.

[5] S. Aharinejad and A. Lametschwandtner, Microvascular Corrosion Casting in Scanning

Electron Microscopy: Techniques and Applications, Springer, New York, 1992. 
TABLE 1. Number and size of the specimens, and the valvular density per region.

* number of valves per specimen, $* *$ specimen size, $* * *$ calculated valvular density $/ \mathrm{cm}^{2}$

\begin{tabular}{|c|c|c|c|c|c|c|c|c|}
\hline Specimen & $\mathbf{1}$ & $\mathbf{2}$ & $\mathbf{3}$ & $\mathbf{4}$ & $\mathbf{5}$ & $\mathbf{6}$ & $\mathbf{7}$ & $\mathbf{8}$ \\
\hline Region 1 & $0 * / 128^{* *}$ & $7 / 180$ & $3 / 130$ & $0 / 96$ & $1 / 112$ & $0 / 36$ & $0 / 52$ & $6 / 1.54$ \\
(rect.abd.sup.) & $0 * * *$ & 3.89 & 2.31 & 0 & 0.89 & 0 & 0 & 3.9 \\
\hline Region 2 & $2 / 169$ & $0 / 57$ & $0 / 70$ & $1 / 180$ & $1 / 26$ & $1 / 126$ & $0 / 72$ & $0 / 99$ \\
(rect.abd.med.) & 1.18 & 0 & 0 & 0.56 & 3.85 & 0.79 & 0 & 0 \\
\hline Region 3 & $3 / 156$ & $5 / 216$ & $0 / 72$ & $0 / 24$ & $3 / 128$ & $1 / 96$ & $1 / 156$ & $0 / 153$ \\
(rect.abd.inf.) & 1.92 & 2.31 & 0 & 0 & 2.34 & 1.04 & 0.64 & 0 \\
\hline Region 4 & $3 / 150$ & $3 / 133$ & $9 / 323$ & $2 / 304$ & $1 / 63$ & $1 / 143$ & & \\
(lat.dorsi sup.) & 2.0 & 2.26 & 2.79 & 0.66 & 1.59 & 0.7 & & \\
\hline Region 5 & $3 / 150$ & $0 / 77$ & $4 / 225$ & $2 / 220$ & $3 / 168$ & $3 / 132$ & & \\
(lat.dorsi med.) & 2.0 & 0 & 1.78 & 0.91 & 1.79 & 2.27 & & \\
\hline Region 6 & $0 / 18$ & $0 / 78$ & $0 / 9$ & $2 / 70$ & $0 / 252$ & $1 / 221$ & $4 / 238$ & $0 / 224$ \\
(lat.dorsi inf.) & 0 & 0 & 0 & 2.86 & 0 & 0.45 & 1.68 & 0 \\
\hline
\end{tabular}
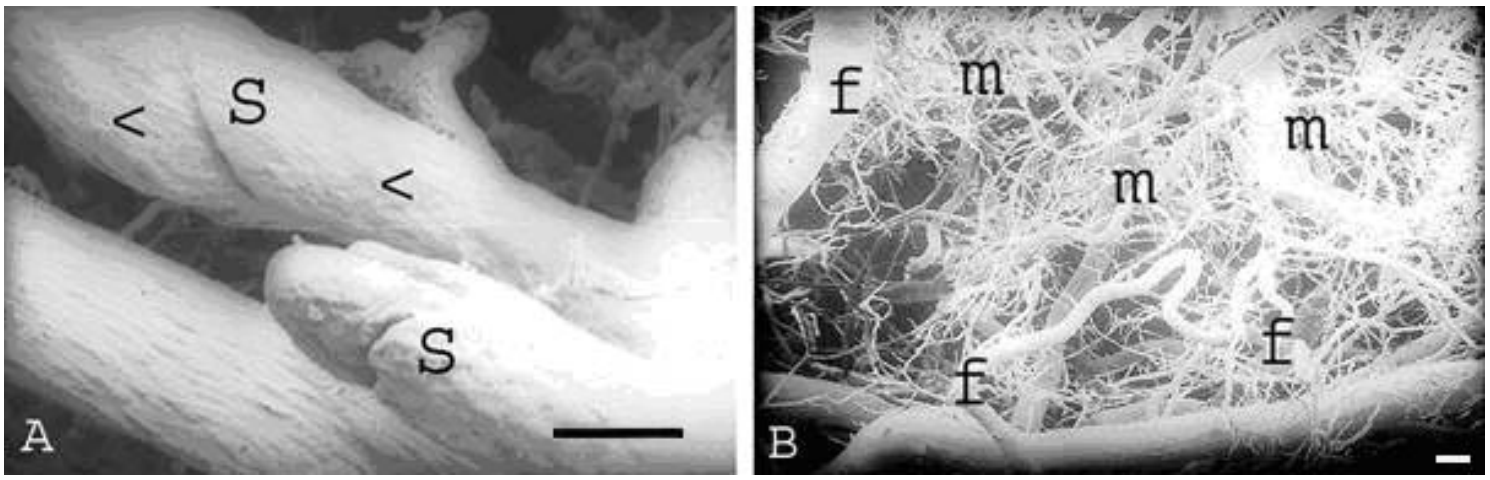

Fig. 1. A. Two smaller venous casts with bicuspid valves. "S" marks valvular sinus. Endothelial cell nuclear imprints (arrowheads) are visible as roundish structures. The veins were found in the latissimus dorsi muscle beneath the muscle fascia. Bar $=50 \mu \mathrm{m}$. B. The image shows the border zone between the rectus abdominis muscle fascia (" $\mathrm{f}$ ") and the deeper muscular areas ("m") where all valves were found. Bar $=200 \mu \mathrm{m}$.

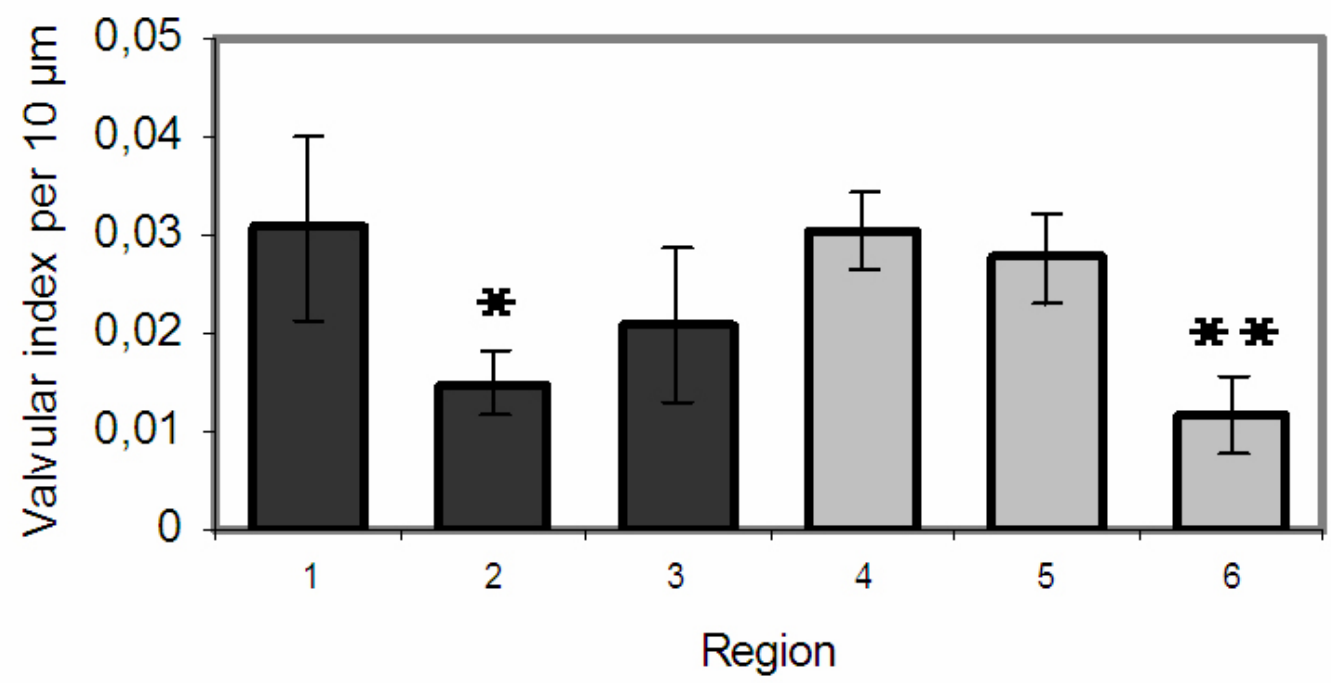

Fig. 2. The diagram shows the calculated valvular index per $10 \mu \mathrm{m}$ venous length for the regions 1-6 in both the rectus abdominis (regions 1-3) and latissimus dorsi (regions 4-6).

* marks significance compared to region $1 ; * *$ marks significance vs. regions 4 and 5. 